This is an Open Access article distributed under the terms of the Creative Commons Attribution-Noncommercial License, which permits unrestricted use, distribution, and reproduction in any noncommercial medium, provided the original work is properly cited.

\title{
CHARACTERIZING EXTRA-SOLAR PLANETS WITH COLOR DIFFERENTIAL ASTROMETRY ON SPICA
}

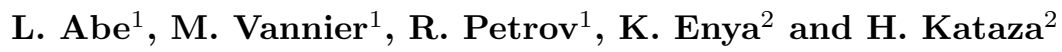 \\ ${ }^{1}$ Laboratoire Hippolyte Fizeau, UMR6525, Universite de Nice Sophia-Antipolis, 28, avenue Valrose, 06108 Nice Cedex 02 , \\ France \\ ${ }^{2}$ Institute of Space and Astronautical Sciences, Japan Aerospace Exploration Agency, Yoshinodai 3-1-1, Sagamihara, \\ Kanagawa 229-8510, Japan
}

\section{ABSTRACT}

SPICA coronagraph will be operating from 3.5 to 27 micron and primarily aims at directly imaging extra-solar planets of nearby stars. A strong limitation of the coronagraph for the $3.5 \mathrm{~m}$ telescope is its angular resolution which allows planet detectability farther than a few AUs at best. Color Differential Astrometry (CDA) is a spectrophotometric method which would push the detection range to very close-in planets and up to a few AUs, thus nicely complementing the imaging coronagraph. We present the method and show how it can be implemented within the host coronagraph without major impacts on its optical setup. We give preliminary estimates of its sensitivity according to different extra-solar planetary systems configurations. Critical instrumental requirements are discussed, especially regarding detector gain stability.

Key words: Stars: formation - Missions: SPICA

\section{INTRODUCTION}

The SPICA mission will cover a wide spectral range from NIR to sub-millimeter wavelengths. With a nominal cooling temperature of $4.5 \mathrm{~K}$ for the primary optics and most of the instruments, SPICA will reach outstanding sensitivity at longer wavelengths (i.e. MIR and above). One of the science goals of SPICA is to investigate the formation and evolution of planetary systems through the study of circumstellar discs, and of course through the direct observation and characterization of planets. SPICA is foreseen to be equipped with a coronagraph for high contrast direct imaging of planets, with a goal contrast of at least $10^{6}$ at the lowest possible angular distance from their parent star. However, the direct imaging angular resolution is intrinsically limited by the well known diffraction limit $\lambda / \mathrm{D}$ which, for SPICA, corresponds to about 0.2 arcsec at its shortest wavelength $(3.5 \mu \mathrm{m})$, about $2 \mathrm{AUs}$ at a distance of $10 \mathrm{pc}$. In this paper we present a revised concept of the Color Differential Astrometry mode (Vannier et al., 2003), free from calibration devices (i.e. moving parts) that are undesirable with such a space telescope.

\section{Color Differential Astrometry}

Color Differential Astrometry is a technique that has been used in combination with several speckle interferometry techniques, on monolithic telescopes and on diluted aperture interferometers (Chelli \& Petrov, 1995). The primary goal of the method is to recover both astrometric and spectral data from a system whose configuration is known $a$ priori.

\subsection{Photocenter displacement}

It has been well established that the relative position of an image can be measured with a precision much higher than its equivalent size (Beckers, 1981). In the present case, the position refers to the photocenter, i.e. the barycenter of the brightness distribution $o(\boldsymbol{r}, \lambda)$ of the source object at angular position $r$ and at wavelength $\lambda$. Consider the case where the target consists of two sources (assumed to be punctual), such as a star and an extrasolar planet with respective spectral energy distributions $s_{\star}(\lambda)$ and $s_{p}(\lambda)$ ). Let $s(\lambda)=s_{p}(\lambda)+s_{\star}(\lambda)$ be the spectrum of the star+planet system, and $\boldsymbol{\rho}(t)$ their angular separation at time $t$. The position of the photocenter $\boldsymbol{\epsilon}_{\boldsymbol{s}}(\lambda, t)$ is :

$$
\boldsymbol{\epsilon}_{\boldsymbol{s}}(\lambda, t)=\frac{\int \boldsymbol{r} o(\boldsymbol{r}, \lambda) d \boldsymbol{r}}{\int o(\boldsymbol{r}, \lambda) d \boldsymbol{r}}=\boldsymbol{\rho}(t) \frac{s_{p}(\lambda)-s_{\star}(\lambda)}{s(\lambda)}+\boldsymbol{c}
$$

Where $\boldsymbol{c}$ is a constant vector which depends on the spatial origin of the position $\boldsymbol{r}$. If the origin is chosen on the star, and considering that $s_{p}(\lambda) \ll s_{\star}(\lambda)$, then eq.1 can be approximated by:

$$
\boldsymbol{\epsilon}_{\boldsymbol{s}}(\lambda, t)=\boldsymbol{\rho}(y) \frac{s_{p}(\lambda)}{s(\lambda)}
$$

\section{RECOVERING PLANET POSITION AND SPECTRUM}

The photocenter measurement in eq.2 yields a mixed information on the separation vector and on the luminosity ratio $s_{p}(\lambda) / s(\lambda)$. If $\boldsymbol{\rho}(t)$ is known, the joint measurement of $\boldsymbol{\epsilon}_{\boldsymbol{s}}(\lambda, t)$ by CDA and of $s(\lambda)$ by spectroscopy trivially yields the star and planet spectra $s_{\star}(\lambda)$ and $s_{p}(\lambda)$ without any further hypothesis on $s_{\star}(\lambda)$. This can occur, for instance, when $t$ corresponds to a maximum angular separation, which is known in the case where the spectroscopic orbit is measured using the radial velocity method. 
In the general case where $\boldsymbol{\rho}$ is unknown, several observations at different orbital phases are necessary to separate the spectral and orbital variables. The necessary number of measurements of vector $\boldsymbol{\epsilon}_{\boldsymbol{s}}$ depends on the information we have from the spectroscopic orbit, and may range from 3 to 6 . If only one component of $\epsilon_{\boldsymbol{s}}$ is observed at a given time (i.e. the photocenter displacement is projected along one axis only), the total number of measures has to be doubled. Finally, the number of measurements can be further increased if taking into account the time-dependency of the planetary spectrum, due to the variable difference of re-emitted flux between the "day" and "night" sides of the planet, along the orbital phase. However, this further degree of complexity would be only relevant for the most irradiated "pegasi-like" giant planets.

\section{NumericAl MODELS AND RESUlts}

\subsection{Atmosphere models}

For the planetary spectra we used updated versions of the models presented by Barman et al. (2001) and Barman et al. (2003). They describe the atmosphere of giant gaseous planets under high stellar irradiation, and consider two possibilities : a "condensed" model, where all the atmospheric dust has settled out, and a "dusty" one, with clouds of dust still suspended in the atmosphere, thus contributing to its opacity. In the present work we use the first model, which is favored by these authors. Without any atmospheric opacity, the impinging radiation alters the inner layers of the planet and tends to amplify the spectral features at short wavelengths. We considered an intrinsic temperature of $500 \mathrm{~K}$.

\subsubsection{FUndAMENTAL NOISES}

The fundamental noises that we consider here are photon noise, thermal noise (from the telescope and from the instrument), zodiacal light, and detector read-out noise. If we focus on the case of nearby, relatively bright stars, on which observations of extrasolar planets should be targeted, the noise budget is clearly dominated by the photon noise $1 / \sqrt{\mathrm{N}_{\text {phot }}}$. The number of collected photons is determined, at a given wavelength, by the apparent stellar magnitude $(m)$, the exposure time $(T)$, the transmission of the instrument optics $\left(t_{i}\right)$ and the spectral resolution $(R)$. The error on one component (i.e. coordinate) of the photocenter position $\epsilon$ from the fundamental noise is therefore given by the relation:

$$
\sigma_{\epsilon} \approx \frac{1}{\sqrt{2 \mathrm{~N}_{\mathrm{phot}}}} \propto 10^{-0.2 m} \sqrt{\frac{R}{2 t_{i} T}}
$$

\subsection{Potential SNR And SCientific CASes}

Based on the planetary atmosphere and fundamental noise models described above, we performed a few simulations in order to explore the observation potential along the following parameters: stellar spectral types $\mathrm{G}^{*}$ and $\mathrm{M}^{*}$, planetary orbital separation, and distance of the system. The signal-to-noise ratios described hereafter do not take into account the limits from instrumental stability, which are discussed in the next section. The reference channel for the differential measurement was arbitrarily taken at the shortest wavelength; some other choice would of course change the shape of the signal amplitude, although it would globally remain at a comparable level.

Around a solar-type star, the signal stands clearly above the fundamental noise level for planets with an orbital separation ranging from 0.05 to $1 \mathrm{AU}$ (Fig. 1). The closest separations favor the signal at short wavelength (where the higher temperature of the planet compensates the smaller angular separation in the photocenter displacement), whereas the largely separated planets are better measured towards longer wavelength. Also, planets with not-so-short separations show significantly larger variations of the signal over the spectrum, which might offer interpretation in terms of atmospheric composition and physical characteristics.

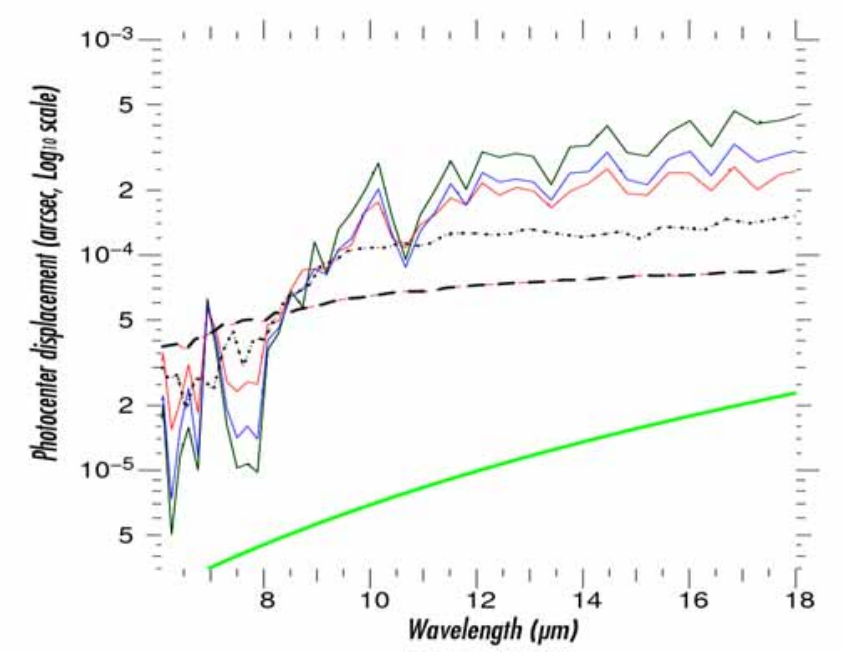

Figure 1. Expected signal and fundamental noise levels for a G2V star at $5 \mathrm{pc}$, and an irradiated planet orbiting at (from bottom to top) $0.05 \mathrm{AU}$ (dashed), $0.1 \mathrm{AU}$ (dotted), $0.3 \mathrm{AU}$ (red), $0.5 \mathrm{AU}$ (blue) and $0.1 \mathrm{AU}$ (black). The spectral resolution is $R=$ 40 , and the fundamental noise (thick green line at the bottom) corresponds to an exposure of 2 hours.

At a given orbital separation, the signal-to-noise ratio varies roughly with the squared distance of the system, due to the combined effect of the smaller angular separation and increased photon noise with distance. This is illustrated in Fig. 2: only systems located shorter than 
10 parsec can be observed with a decent SNR, for the parameters used in these simulations.

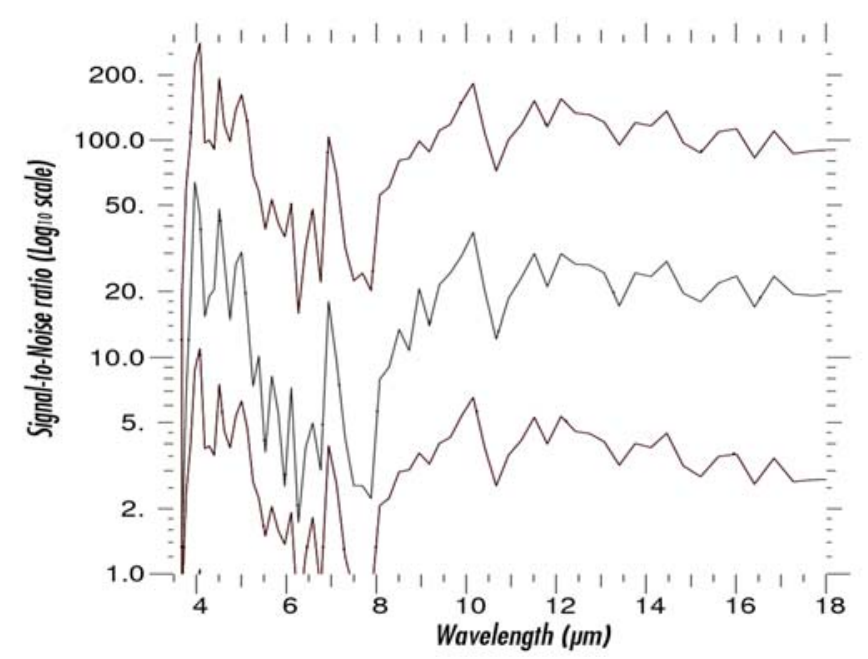

Figure 2. Expected Signal-to-noise ratio for an irradiated planet orbiting at $0.5 \mathrm{AU}$ around a G2V star, located at distances of 2, 5 and 10 parsec (from top to bottom). The spectral resolution is $R=40$ and the exposure time is 2 hours

We also simulated the case of a planet around a closeby red dwarf (M5) star. Figure 3 shows that, although less favourable than with a solar-type star, a possible detection is not excluded: at a distance of 5 parsec, a SNR of a few tens can be attained for planets separated by $0.2 \mathrm{AU}$ or $0.5 \mathrm{AU}$.

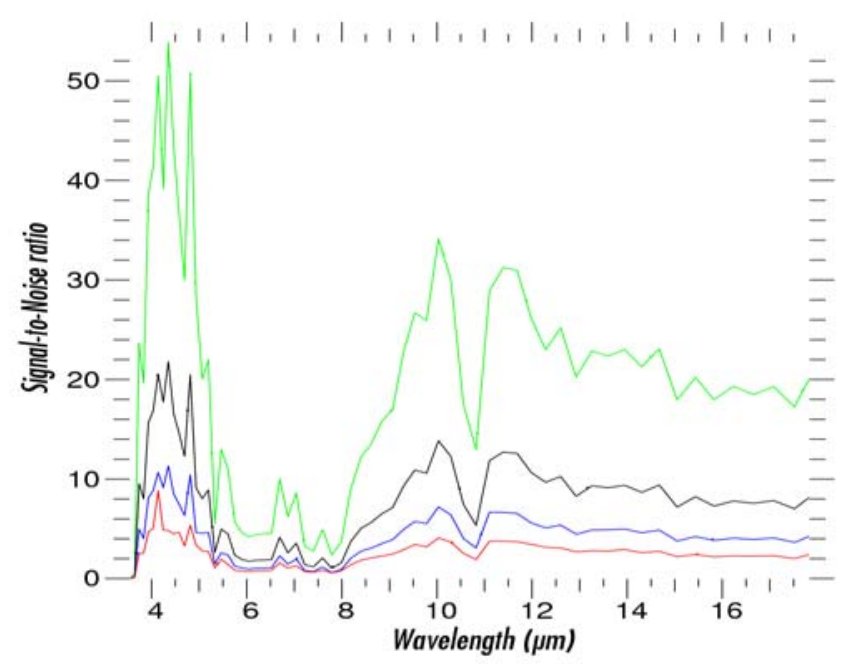

Figure 3. Expected signal-to-noise ratio for an M5 star at $5 \mathrm{pc}$, and an irradiated planet orbiting at (from bottom to top) $0.05 \mathrm{AU}$ (red), $0.1 \mathrm{AU}$ (blue) $0.2 \mathrm{AU}$ (black) and $0.5 \mathrm{AU}$ (green). The spectral resolution is $R=40$ and the exposure time is 2 hours.
From these examples, the scientific case for differential astrometry onboard SPICA can therefore be summarized as following. It consists in doing low-to-medium resolution spectroscopy, and to derive the orbital parameters of:

- Planets already detected (using velocimetry techniques) around solar-type stars at a distance up to 10 parsec, with orbital separations ranging from $0.05 \mathrm{AU}$ to $0.1 \mathrm{AU}$.

- Planets around later-type main sequence stars (up to $\mathrm{M}$ type), for distances smaller than 10 parsec and orbital separations larger than 0.1 AU. For a given separation, the SNR will decrease with the spectral-type, therefore requiring longer exposure times for the reddest stars.

\subsection{Instrumental Limitations and Calibration}

\subsubsection{SATURATION AND NON-LINEARITY}

Saturation or non-linearity of the measurement might be problematic, given that the target sources are quite close and that the detector can probably not be read-out in less than one second. This critical issue will depend on some still-pending choices on the optical design. In particular, saturation may well occur if the PSF is fully anamorphized in the direction of spectral dispersion so that, for a given spectral channel, the flux is summed along a line of pixels. Considering a read-out time of $2.7 \mathrm{~s}$, and a global instrumental throughput of $50 \%$ we find that the maximum photon count of an anamorphized PSF from a solar-type star at 10 parsec, spread over 12 pixels and with a spectral resolution $R=40$, would be about 300000 . This would then exceed the full-well capacity (which we estimate to be at about $250000 \mathrm{e}^{-}$). If these assumptions are correct, a flux density of at least 10 would be necessary for observing $G$ (or later) type stars down to a few parsecs of distance.

On the other hand, a partially or non-anamorphized PSF would relax the saturation constraint, since the flux would be spread along various pixels in the spectral direction. Assuming that the photon count stays below the saturation limit, the effects possibly introduced by nonlinearity are still to be studied.

\subsubsection{Detector Gain FluCtuations}

The observation calibration requires pointing an off-axis reference star. We expect that the optical path will not change dramatically between the two observations. The main factor affecting the calibration process is expected to be the variation of gain table, combined to the pointing error: the photocenters from of science and the calibrator sources will be measured on pixels with slightly different gains. We have performed simulations to measure that effect, assuming a few hypotheses which seem compatible with the chosen detector: global (i.e. uniform) gain variation of $1 \%$ and pointing offset of 0.3 pixels (i.e. 
$\sim 20$ mas) between the reference calibrator and the science star. In addition, the detector gain table varies with a rugosity (i.e. noise-like) of up to $2 \%$. Our computation (Fig. 4) shows that the error on the measurement is of the order of $10^{-5}$ arcsec for the largest variation of gain. This represent a significant degradation (especially at shortest wavelength) with respect to the fundamental SNR, but still allows a detection in several of the cases presented above. The most problematic cases will be the planets at the largest orbital separations (i.e. > 0.5 AU), for which the signal is lower at short wavelength, and the measurement of systems around late-type stars between 5 and $8 \mu \mathrm{m}$.

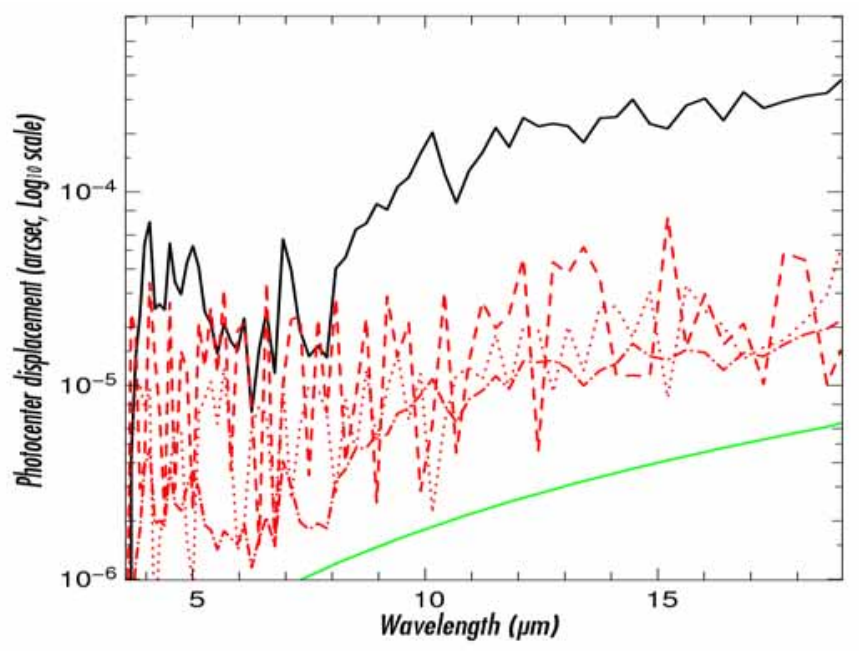

Figure 4. Expected signal and noise levels for a G2V star at $5 \mathrm{pc}$, and an irradiated planet orbiting at $0.5 \mathrm{AU}$. The pointing offset between the reference and the science star is set to 0.3 pixels. The detector exposure time is 2 hours while the gain table global offset between the reference and science star is $1 \%$. The dash-dotted, dotted and dashed correspond to a detector gain table rugosity of $0,0.5$ and $2 \%$ respectively (see text for details about noise sources).

\section{Practical Considerations}

The SPICA telescope will be actively cooled in orbit using mechanical cryo-coolers. The induced vibrations by these mechanisms are likely to destroy our ability to precisely measure the photocenter position. However, the most important thing is probably, rather than the actual jitter amplitude itself, the low-trend stability over time (absence of drifts) of the pointing direction: since we are using a calibration star, we need the science and calibration stars to ideally fall on the exact same pixels in order to minimize the effect of pixel gain differences between the two sources (see below).

In order to prevent pointing drifts and to reduce the telescope jitter - about 1 arcsec (Enya, 2009) - we rely on the coronagraph tip-tilt mirror, which is currently under development at the Institute for Astronomy, University of Tokyo (Nakamura et al., 2008; Miyata et al., 2008).

\section{Conclusion}

In this paper we showed that from fundamental SNR calculations, there is a potential for CDA to perform low-tomedium resolution spectroscopy of planets orbiting at a fraction of AU around nearby ( $<10$ parsecs) main-sequence stars.

The initial observing strategy in the context of the SPICA mission would be to derive atmospheric characteristics for the planets already detected indirectly through radial velocity methods. A secondary objective might also consist in a "blind exploration" mode for targets of interest, for example in the case of red dwarves where giant planets may be orbiting farther away, beyond the current radial velocity technique sensitivity. This type of targets might be directly detected by coronagraphic imaging, or searched/confirmed by CDA observations.

The technical scheme for CDA seems to be compatible with current design and constraints of SPICA coronagraph since we only rely on a low to medium spectroscopic mode, without any moving parts, as proposed in earlier concepts for CDA calibration. We only require that mainly the detector is very stable over time. However, several technical issues need further investigation: saturation and non-linearity, more complex detector instabilities, etc... Although CDA has already been used in speckle interferometry observations, we plan to demonstrate accurate photocenter displacement at a level better than $1 / 100$ th of a pixel, in the visible range at first, then extrapolate these results to refine our requirements for the detector.

\section{REFERENCES}

Barman, T. S., Hauschildt, P. H., \& Allard, F. 2001, ApJ, 556,885

Barman, T. S., Allard, F., Baraffe, I., Chabrier, G., \& Hauschildt, P. H. 2003, Bulletin of the American Astronomical Society, 35, 1234

Beckers, J. M. 1981, Lowell Observatory Bulletin, 9, 165

Chelli, A., \& Petrov, R. G. 1995, A\&A Supplement Series, 109,389

Enya, K. for the SPICA Working Group 2009, arXiv, 0905.3829

Miyata, T., Sako, S.,Nakamura, T., Onaka, T., \& Kataza, H. 2008, SPIE Proc., 7014,

Nakamura, T., Miyata,T., Sako, S., Onaka, T., Enya, K., Kataza, H., Takahashi, H., \& Obuchi, Y. 2008, SPIE Proc., 7018,

Vannier, M., Petrov, R. G., Bensammar, S. \& Lopez, B. 2003, EAS Publications Series, Vol. 8, Astronomy with High Contrast Imaging, Proc. of the conference held 1316 May, 2002 in Nice, France, pp. 171-180. 